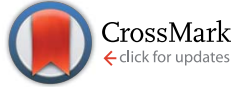

Cite this: Soft Matter, 2014, 10, 7712

\title{
Microgel-like aggregates of isotactic and atactic poly(methacrylic acid) chains in aqueous alkali chloride solutions as evidenced by light scattering $\dagger$
}

\begin{abstract}
Simona Sitar, ${ }^{a}$ Vladimir Aseyev ${ }^{\mathrm{b}}$ and Ksenija Kogej ${ }^{* a}$
A comparative light-scattering study of isotactic and atactic poly(methacrylic acid), iPMA and aPMA, respectively, in aqueous solutions with added alkali chlorides, $\mathrm{XCl}\left(\mathrm{X}=\mathrm{Li}, \mathrm{Na}, \mathrm{Cs}\right.$ ), at $25{ }^{\circ} \mathrm{C}$ and $\mathrm{XCl}$ concentration of $0.1 \mathrm{~mol} \mathrm{~L}^{-1}$, demonstrates that both PMA isomers are strongly associated at low degrees of neutralization, $\alpha_{N}(=0$ for aPMA and 0.25 for iPMA), in the presence of all XCls. The shape parameter $\rho$ and the scattering functions suggest that aggregates have the characteristics of microgel particles, with a dense core surrounded by a less dense shell. The extent of aggregation depends on the stereoregular structure of the polymer and on the type of the added cation. $\mathrm{Li}^{+}$and $\mathrm{Na}^{+}$ions support aggregation better than $\mathrm{Cs}^{+}$ions. Besides, iPMA chains are more strongly aggregated than aPMA chains and form particles with a denser core. A model of the aggregation process is suggested for iPMA. At high $\alpha_{N}$, a slow diffusive process (so-called extraordinary or anomalous mode in diffusion of polyelectrolytes), arising from electrostatic interactions between charged chains, is observed for both PMAs. Results suggest that under the same experimental conditions iPMA is effectively more charged than aPMA. The role of ions in the slow-mode phenomenon is less pronounced than in aggregation.
\end{abstract}

Received 2nd July 2014
Accepted 29th July 2014

DOI: $10.1039 / c 4 s m 01448 k$

www.rsc.org/softmatter

\section{Introduction}

Polyelectrolytes often self-organize into large-scale structures by electrostatic and other forces that can be directed by various parameters, e.g. by polyion charge, the presence of other charged species in solution (from small and more mobile ions to surfactant ions and colloids), temperature, solvent, etc. The ability to control intermolecular association between polyelectrolyte chains is of great importance in nature and in various applications. A recent themed issue of Soft Matter on polyelectrolytes in biology and soft matter ${ }^{1}$ discusses the current state of the art in this field from various aspects. Although natural polyelectrolytes (DNA and RNA) have been a focus of research in recent years, synthetic polyelectrolytes with simpler structures still help to identify mechanisms on the molecular level and aid in understanding complex phenomena.

Among synthetic polyelectrolytes, the weak types, like poly(methacrylic acid), PMA, ${ }^{2}$ are good candidates for studying aggregation/association phenomena, due to the fact that their charge can easily be varied by changing the $\mathrm{pH}$ of the medium.

${ }^{a}$ Department of Chemistry and Biochemistry, Faculty of Chemistry and Chemical Technology, University of Ljubljana, Aškerčeva 5, P.O. Box 537, SI-1001, Ljubljana, Slovenia.E-mail: ksenija.kogej@fkkt.uni-lj.si

${ }^{b}$ Laboratory of Polymer Chemistry, Department of Chemistry, University of Helsinki, P.O. Box 55, FIN-00014 HU, Helsinki, Finland

† Electronic supplementary information (ESI) available. See DOI: $10.1039 / \mathrm{c} 4 \mathrm{sm} 01448 \mathrm{k}$
Furthermore, mere variation of the stereochemical structure of the polymer chain may have a strong effect on intermolecular interactions, aggregation, and solution properties in general. ${ }^{3}$ PMA is one of the simplest synthetic polymers for which solution and aggregation behaviors depend significantly on the chain tacticity. ${ }^{2,4-7}$ It has been demonstrated, for example, that the $\mathrm{pH}$-induced conformational transition is reversible for socalled conventional or atactic PMA, aPMA, ${ }^{2,8}$ but irreversible for the structurally highly ordered isotactic PMA, iPMA. ${ }^{4,8}$ The underlying molecular reasons for such behavior are far from being satisfactorily explained.

In the past, research interest in PMA was mostly related to the fact that the PMA chain adopts a compact conformation at low $\mathrm{pH}$ (low degrees of neutralization, $\alpha_{\mathrm{N}}$ ) and undergoes a conformational transition upon increasing $\mathrm{pH}$ (i.e. $\alpha_{\mathrm{N}}$ ), which takes place on the intramolecular level. Less attention, however, was devoted to the ability of PMA to form intermolecular aggregates in aqueous solutions at low $\alpha_{\mathrm{N}}$, even though Eliassaf et al.,10 demonstrated already in the 1960s that the unneutralized aPMA $\left(\alpha_{\mathrm{N}}=0\right)$ is capable of forming gel networks in concentrated aqueous solutions. Gel formation was induced either by heating ${ }^{9}$ or shearing $^{\mathbf{1 0}}$ of solutions. The interpretation of the shearing-induced association was that physical crosslinks between the aPMA chains are more likely to be formed in flow than in a system at rest, as demonstrated both in concentrated ${ }^{\mathbf{1 0}}$ and later also in more dilute conditions. ${ }^{\mathbf{1 1 - 1 3}}$ On the other hand, the structurally more ordered iPMA has received considerably less attention, although it exhibits some very 
obvious differences in comparison with aPMA. In addition to the above-mentioned irreversible conformational transition, iPMA is insoluble in water when $\alpha_{\mathrm{N}}<\sim 0.2$ (ref. 5, 6 and 14) and exhibits a high tendency to form aggregates in aqueous solutions. ${ }^{6,7,13}$ Van den Bosch et al. ${ }^{6}$ have demonstrated that a 10 $\mathrm{w} / \mathrm{w} \%$ iPMA solution with $\alpha_{\mathrm{N}}=0.28$ very rapidly forms an elastic gel when cooled below $0{ }^{\circ} \mathrm{C}$, whereas an opposite behavior was reported for aPMA: ${ }^{9}$ a sample of aPMA $\left(\alpha_{N}=0\right)$ with a rather high molar mass formed a gel network in concentrated solution when heated above $47^{\circ} \mathrm{C}$. Pronounced intermolecular association was also revealed by studies of very dilute iPMA solutions. ${ }^{7}$

It is well known that the properties of polyelectrolytes depend also on the nature of the ions that are present in solution. The effect of ions on the properties of proteins was recognized more than 100 years ago by Hofmeister ${ }^{15,16}$ who ranked ions in a specific series (the Hofmeister series) according to their ability to induce precipitation of proteins from water. Collins ${ }^{17-19}$ proposed a simple law of matching water affinities, LMWA, which relates these phenomena to the tendency of oppositely charged ions (groups) to form so-called contact ion pairs. These are formed if the affinity of oppositely charged ions for water, manifested through the ion's hydration enthalpy, is similar. ${ }^{17,19-22}$ In the case of polyelectrolytes, the interaction of simple ions with charged macromolecular surfaces has to be considered. Thus, in relation to PMA, Strauss $^{23}$ showed that volume changes accompanying interactions of PMA with alkali metal ions depend on the ion's hydration radius. In line with the LMWA proposed later, this was attributed to the competition between hydrating water molecules and the PMA's carboxylate groups for a given cation. However, the ion-specific effects were, until now, not investigated systematically with PMA, in particular not in relation to chain tacticity. Since the carboxylate functional group is widespread in biology and in colloid chemistry, PMA may serve as a study example of the effect of ions on the stability and structure in solutions of such macromolecules.

In this contribution, we investigate the effect of alkali metal ions on aggregation/association phenomena in aqueous PMA solutions. We present a static, SLS, and dynamic light-scattering, DLS, study of both PMA isomers, isotactic and atactic, in dilute aqueous solutions at $25{ }^{\circ} \mathrm{C}$ and with increasing $\alpha_{\mathrm{N}}$ in the presence of $\mathrm{LiCl}, \mathrm{NaCl}$, and $\mathrm{CsCl}$. The main objectives of the study are to identify the nature of the aggregates between PMA chains and the function of alkali metal cations in aggregation (the low $\alpha_{\mathrm{N}}$ case), and additionally to reveal their role in intermolecular interactions with increasing polyion charge (the higher $\alpha_{\mathrm{N}}$ cases). In order to achieve the first objective we determine the hydrodynamic radius, $R_{\mathrm{h}}$, and when possible the radius of gyration, $R_{\mathrm{g}}$, of particles in solution and, from these, their shape parameter, $\rho\left(=R_{\mathrm{g}} / R_{\mathrm{h}}\right)$. To realize the second goal, light-scattering, LS, studies are extended to higher $\alpha_{\mathrm{N}}$ values (up to $\alpha_{\mathrm{N}}=1$ ) where electrostatic repulsion between polyions prevents aggregate formation, but leads to so-called anomalous diffusion of polyions (a diffusive mode in DLS). This phenomenon is described by various terms in the literature, as an extraordinary mode, ${ }^{24}$ slow mode, ${ }^{25,26}$ polyelectrolyte effect, ${ }^{27}$ or multimacroion domain or cluster formation, ${ }^{25,28-31}$ and still presents a major challenge in investigations of polyelectrolyte behavior in solutions. The slow mode stems from electrostatic repulsion between similarly charged polyions and manifests itself as an apparent diffusion coefficient in correlation functions measured by DLS. Therefore, we will use the term slow mode for this feature within this manuscript. We address all these issues in relation to chain tacticity.

\section{Experimental section}

\section{Materials}

aPMA (Polymer Source, Inc., sample no. P14096-MAA, with weight and number average molar masses $M_{\mathrm{w}}=189000 \mathrm{~g}$ $\mathrm{mol}^{-1}$ and $M_{\mathrm{n}}=165000 \mathrm{~g} \mathrm{~mol}^{-1}$, respectively $)$ and iPMA $\left(M_{\mathrm{w}}=\right.$ $32000 \mathrm{~g} \mathrm{~mol}^{-1}, M_{\mathrm{n}}=11000 \mathrm{~g} \mathrm{~mol}^{-1}$ ) were the same as used previously. ${ }^{13}$ The stereoregular composition of both PMAs was determined from the NMR spectrum of the methylated forms of polyacids. The aPMA sample is rich in syndiotactic triads and the iPMA sample contains around 93\% of isotactic triads. The stereoregular compositions of both PMAs are reported in Table S1 (ESI $\dagger$ ).

$\mathrm{LiCl}, \mathrm{NaCl}$ and $\mathrm{CsCl}$ (all from Merck $\mathrm{KGaA}$ ) were used to prepare aqueous $0.1 \mathrm{M} \mathrm{XCl}$ solutions. Solid LiOH monohydrate (Fluka Biochemica), aqueous $\mathrm{NaOH}$ (Fixanal, Merck KGaA) and aqueous $\mathrm{CsOH}$ (Riedel-de Haen) were used to prepare $\mathrm{LiOH}$, $\mathrm{NaOH}$ and $\mathrm{CsOH}$ solutions, respectively. The concentration of all hydroxide solutions was determined by neutralization potentiometric titration using a standardized $\mathrm{HCl}$ solution.

\section{Preparation of solutions}

aPMA is soluble in water at any $\alpha_{\mathrm{N}}$, whereas iPMA is insoluble unless $\approx 20 \%$ of $\mathrm{COOH}$ groups are ionized. The critical value of $\alpha_{\mathrm{N}}$, denoted as $\alpha_{\mathrm{N}, \text { crit }}$, at which iPMA dissolves in water at $25{ }^{\circ} \mathrm{C}$ is $\alpha_{\mathrm{N}, \text { crit }} \approx 0.2 .^{5,6,14}$ Different procedures were therefore used to prepare aPMA and iPMA solutions with various $\alpha_{\mathrm{N}}$ values, as reported previously ${ }^{\mathbf{1 3}}$ and described in detail in ESI. $\dagger$ For setting the $\alpha_{\mathrm{N}}$ of PMAs, the $\mathrm{XOH}$ solution with the same cation as in the $0.1 \mathrm{M}$ XCls (X) was used.

\section{Methods}

Light scattering. LS experiments were conducted using the 3D cross-correlation spectrometer from LS Instruments $\mathrm{GmbH}$ (Fribourg, Switzerland), which is based on 3D technology specially designed to filter out multiple scattering from the total scattering. ${ }^{32}$ For this purpose, two coherent incident light beams are generated with a $20 \mathrm{~mW}$ He-Ne laser (Uniphase JDL $1145 \mathrm{P}$, operating at $632.8 \mathrm{~nm}$ ) and focused into the same volume in the sample, and the intensity of two scattered beams is measured simultaneously. The instrument is equipped with a laser attenuation system combined with an online incident laser intensity detector that allows for the normalization of the LS intensity (see also ESI $\dagger$ ).

LS measurements were performed in cylindrical quartz cells. Solutions were gently filtered directly into the measuring cell through hydrophilic Millex-HV filters with a pore size of $0.22 \mu \mathrm{m}$ and a diameter of $13 \mathrm{~mm}$. The initial few $\mu \mathrm{L}$ of the filtered 
solution were discarded. No significant loss of the polymer was observed due to filtration, which was verified by checking the intensity of scattered light from unfiltered and filtered solutions. The samples were allowed to equilibrate for $20 \mathrm{~min}$ before the measurement was initiated. The intensity of scattered light was collected in the angular range from $40^{\circ}$ to $150^{\circ}$. All LS studies were performed at $25{ }^{\circ} \mathrm{C}$.

Methodological aspects of DLS and SLS can be found elsewhere. $^{33,34}$ At low $\alpha_{\mathrm{N}}\left(\alpha_{\mathrm{N}}=0\right.$ and 0.25 for aPMA and iPMA, respectively), the measured correlation functions were analyzed with the program CONTIN and two well-separated peaks were obtained in the distribution of the correlation times. The calculated distributions were used to split the total scattering intensity into two contributions. The resulting dynamic modes were analyzed individually in order to obtain the corresponding diffusion coefficients (and from those the hydrodynamic radii), and the radii of gyration in the case of large particles. The correlation functions for $\alpha_{\mathrm{N}} \geq 0.4$ (iPMA) and $\alpha_{\mathrm{N}}=1$ (aPMA) also exhibited two dynamic modes, a fast and a slow (extraordinary) mode (for more details see Results and discussion). In iPMA solutions, the relaxation time distributions were overlapping and CONTIN analysis was difficult. A bi-exponential fit was used instead to obtain the relaxation times for both modes. No such problem was encountered with aPMA. Details on all these procedures are described in the literature ${ }^{35,36}$ and in the ESI $\dagger$ accompanying this paper. Examples of data analysis are shown in Fig. S1-S3. $\dagger$ Representative correlation functions are given in Fig. S4a and $b . \dagger$

Determination of the refractive index increment. A Wyatt Optilab rEX differential refractometer (operating at $632.8 \mathrm{~nm}$ ) was used to determine the dependence of the refractive index, $n$, on concentration, $c_{\mathrm{p}}$, from which the refractive index increment, $\mathrm{d} n / \mathrm{d} c_{\mathrm{p}}$, was determined for aPMA and iPMA in aqueous $0.1 \mathrm{M} \mathrm{XCl}$ solutions at $25{ }^{\circ} \mathrm{C}$. The $\mathrm{d} n / \mathrm{d} c_{\mathrm{p}}$ values were measured for $\alpha_{N}$ values in the range 0-1 (aPMA) and 0.25-1 (iPMA) and for $c_{\mathrm{p}}$ values in the range $0.5-4 \mathrm{~g} \mathrm{~L}^{-1}$ (aPMA) and $0.8-8 \mathrm{~g} \mathrm{~L}^{-1}$ (iPMA). The dilutions from the stock solution were performed one day before the measurements were carried out. No effort was put into the determination of the $\mathrm{d} n / \mathrm{d} c_{\mathrm{p}}$ values at a constant chemical potential, $\left(\mathrm{d} n / \mathrm{d} c_{\mathrm{p}}\right)_{\mu}$. In the case of polyelectrolytes, the $\mathrm{d} n / \mathrm{d} c_{\mathrm{p}}$ and $\left(\mathrm{d} n / \mathrm{d} c_{\mathrm{p}}\right)_{\mu}$ values may differ. For example, Schweins et al. $^{37}$ have determined up to $10 \%$ difference (depending on the polymer's $M_{\mathrm{w}}$ ) for sodium poly(acrylate) solutions in $0.1 \mathrm{M} \mathrm{NaCl}$ at $543.5 \mathrm{~nm}$ and $25{ }^{\circ} \mathrm{C}$. However, since our $\mathrm{d} n / \mathrm{d} c_{\mathrm{p}}$ data were used only for the evaluation of the size parameters from the optical contrast of the studied PMA isomers and not for the determination of their absolute $M_{\mathrm{w}}$ values, this difference was ignored.

\section{Results}

\section{The refractive index increment}

The measured $\mathrm{d} n / \mathrm{d} c_{\mathrm{p}}$ values are reported in Table 1 and plotted in Fig. 1 in dependence on $\alpha_{\mathrm{N}}$ for visual appreciation. For aPMA in $0.1 \mathrm{M} \mathrm{LiCl}$ and $\mathrm{NaCl}$ they increase linearly with increasing $\alpha_{\mathrm{N}}$, whereas in $0.1 \mathrm{M} \mathrm{CsCl}$ they decrease in the range $0<\alpha_{\mathrm{N}}<0.5$ and increase for $\alpha_{\mathrm{N}} \geq 0.5$. The highest $\mathrm{d} n / \mathrm{d} c_{\mathrm{p}}\left(=0.193 \mathrm{~mL} \mathrm{~g}^{-1}\right)$
Table 1 The refractive index increment, $\mathrm{d} n / \mathrm{d} c_{\mathrm{p}}$ (in $\mathrm{mL} \mathrm{g}^{-1}$ ), in aqueous iPMA and aPMA solutions at $25^{\circ} \mathrm{C}$ as a function of the degree of neutralization, $\alpha_{\mathrm{N}}$. The concentration of added XCls $(X=N a, L i, C s)$ is $C_{s}$ $=0.1 \mathrm{M}$ and the wavelength is $633 \mathrm{~nm}$

\begin{tabular}{|c|c|c|c|c|c|c|c|c|c|}
\hline \multicolumn{6}{|c|}{ iPMA } & \multicolumn{4}{|c|}{ aPMA } \\
\hline \multicolumn{2}{|l|}{$\mathrm{LiCl}$} & \multicolumn{2}{|c|}{$\mathrm{NaCl}$} & \multicolumn{2}{|c|}{$\mathrm{CsCl}$} & \multirow[b]{2}{*}{$\alpha_{\mathrm{N}}$} & \multirow{2}{*}{$\frac{\mathrm{LiCl}}{\mathrm{d} n / \mathrm{d} c_{\mathrm{p}}}$} & \multirow[t]{2}{*}{$\mathrm{NaCl}$} & \multirow[t]{2}{*}{$\mathrm{CsCl}$} \\
\hline$\alpha_{\mathrm{N}}$ & $\mathrm{d} n / \mathrm{d} c_{\mathrm{p}}$ & $\alpha_{\mathrm{N}}$ & $\mathrm{d} n / \mathrm{d} c_{\mathrm{p}}$ & $\alpha_{\mathrm{N}}$ & $\mathrm{d} n / \mathrm{d} c_{\mathrm{p}}$ & & & & \\
\hline- & & - & & - & & 0 & 0.148 & 0.158 & 0.193 \\
\hline 0.25 & 0.246 & 0.25 & 0.223 & 0.27 & 0.194 & 0.25 & 0.162 & 0.173 & 0.129 \\
\hline 0.35 & 0.256 & 0.34 & 0.208 & 0.36 & 0.183 & - & & & \\
\hline 0.45 & 0.246 & - & & 0.45 & 0.162 & - & & & \\
\hline 0.5 & 0.240 & 0.48 & 0.224 & - & & 0.5 & 0.183 & 0.197 & 0.113 \\
\hline 0.57 & 0.239 & 0.58 & 0.213 & 0.58 & 0.135 & - & & & \\
\hline 0.6 & 0.240 & - & & - & & - & & & \\
\hline 0.75 & 0.265 & 0.75 & 0.219 & 0.75 & 0.147 & 0.75 & 0.201 & 0.214 & 0.137 \\
\hline 1.0 & 0.288 & 1 & 0.229 & 1 & 0.139 & 1.0 & 0.226 & 0.246 & 0.156 \\
\hline
\end{tabular}

at $\alpha_{\mathrm{N}}=0$ is detected in $0.1 \mathrm{M} \mathrm{CsCl}$. For $\alpha_{\mathrm{N}}>0.2$, the ranking of $\mathrm{d} n / \mathrm{d} c_{\mathrm{p}}$ values according to the counterion present in aPMA solutions is $\mathrm{Na}^{+}>\mathrm{Li}^{+}>\mathrm{Cs}^{+}$.

The $\alpha_{\mathrm{N}}$ dependence of the $\mathrm{d} n / \mathrm{d} c_{\mathrm{p}}$ values for iPMA in $0.1 \mathrm{M}$ $\mathrm{CsCl}$ is very similar to the one found for aPMA in $0.1 \mathrm{M} \mathrm{CsCl}$, with a minimum at $\alpha_{\mathrm{N}} \approx 0.5$, and almost constant values for $\alpha_{\mathrm{N}}$ larger than that. In $0.1 \mathrm{M} \mathrm{NaCl}$ and LiCl, however, it is clearly different: in contrast to the linear increase in the whole $\alpha_{\mathrm{N}}$ region in the aPMA case, the $\mathrm{d} n / \mathrm{d} c_{\mathrm{p}}$ values for iPMA are more or less constant $\left(\mathrm{d} n / \mathrm{d} c_{\mathrm{p}} \approx 0.22\right.$ in $0.1 \mathrm{M} \mathrm{NaCl}$ and $\mathrm{d} n / \mathrm{d} c_{\mathrm{p}} \approx 0.24$ in $0.1 \mathrm{M} \mathrm{LiCl}$ for $\alpha_{\mathrm{N}} \leq 0.6$ ) and clearly increase only in $0.1 \mathrm{M} \mathrm{LiCl}$ for $\alpha_{\mathrm{N}} \geq 0.6$. Note also that the ranking of ions is different in comparison with aPMA: the largest $\mathrm{d} n / \mathrm{d} c_{\mathrm{p}}$ values in the whole $\alpha_{\mathrm{N}}$ range are measured in $0.1 \mathrm{M} \mathrm{LiCl}$, leading to the sequence $\mathrm{Li}^{+}>\mathrm{Na}^{+}>\mathrm{Cs}^{+}$for iPMA solutions.

The literature data on the $\alpha_{\mathrm{N}}$ dependence of $\mathrm{d} n / \mathrm{d} c_{\mathrm{p}}$ in aqueous PMA solutions are scarce and reported only for aPMA, sometimes called conventional PMA. Heitz et $a .^{38}$ report a linear dependence in $0.05 \mathrm{M} \mathrm{NaCl}$ and at a lower wavelength (488 nm). The $\mathrm{d} n / \mathrm{d} c_{\mathrm{p}}$ value for the acid form in that work applies to water without added $\mathrm{NaCl}$. This dependence is given as a dashed red line in Fig. 1b. In our case, the line is shifted to lower values, which is expected due to the longer wavelength $(633 \mathrm{~nm})$ of light used in our measurements. ${ }^{39}$ To our knowledge, no literature data on $\mathrm{d} n / \mathrm{d} c_{\mathrm{p}}$ are available for iPMA.

In spite of the fact that the parameter $\mathrm{d} n / \mathrm{d} c_{\mathrm{p}}$ does not affect the determination of $R_{\mathrm{h}}$ and $R_{\mathrm{g}}$, it gives important information on the optical contrast of the studied PMA isomers in aqueous solutions of alkali chlorides. Obviously, the optical contrast depends strongly on the stereoregular structure of PMA and on the type of the added cation. As discussed by Schweins et al., ${ }^{37}$ one may picture polyelectrolyte-simple salt solutions as twophase systems, i.e. domains of polyelectrolyte chains with an ion atmosphere of counterions and co-ions and the polyion-free part of the solution, which contains only the simple (small) ions. The concentration of simple ions is not the same in these domains, which is known as the Donnan effect. Large 


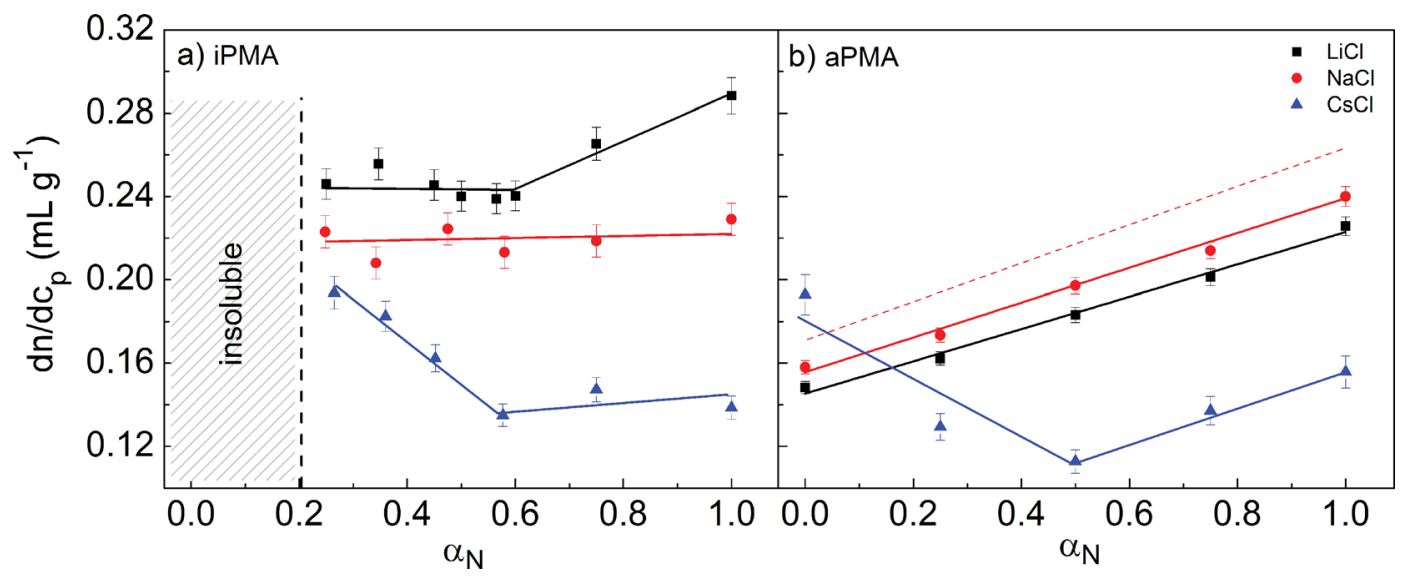

Fig. 1 The dependence of $d n / d c_{p}$ on degree of neutralization, $\alpha_{N}$, in aqueous iPMA (a) and aPMA solutions (b) in the presence of 0.1 M LiCl (black squares), $\mathrm{NaCl}$ (red circles), and $\mathrm{CsCl}$ (blue triangles) at $633 \mathrm{~nm}$. The dashed red line in Fig. $1 \mathrm{~b}$ is based on the equation for the $\mathrm{dn} / \mathrm{d} c_{\mathrm{p}} \mathrm{dependence}$ on $\alpha_{\mathrm{N}}$ of aPMA solutions in $0.05 \mathrm{M} \mathrm{NaCl}$ at $488 \mathrm{~nm}$ as reported. ${ }^{36}$ The solid lines are merely guidance for the eyes.

differences in the $\mathrm{d} n / \mathrm{d} c_{\mathrm{p}}$ dependence on $\alpha_{\mathrm{N}}$ (i.e. on the optical contrast between the polyion domains and the salt-containing solvent) could be explained either by significantly different single chain conformations, and consequently a different distribution of counterions, or by the presence of other species, e.g. aggregates, in solution. In relation to the counterion distribution, it has been shown that the binding of $\mathrm{Na}^{+}$ions is somewhat stronger with iPMA, ${ }^{7}$ but this difference vanishes with decreasing $\alpha_{\mathrm{N}}$. We therefore propose that the most important contribution to the optical contrast in PMA solutions, in particular at low $\alpha_{\mathrm{N}}$ values, is provided by the interchain aggregates. Qualitatively, $\mathrm{d} n / \mathrm{d} c_{\mathrm{p}}$ data suggest that the state of hydrated iPMA chains is different from that of hydrated aPMA chains. This will also become evident from LS results discussed in the following.

\section{Concentration dependence}

The size of single polymer chains in solution generally depends on the polymer concentration. Consequently, we start with a discussion of the effect of $c_{\mathrm{p}}$ on the measured diffusion coefficient, $D$. We first discuss the low $\alpha_{\mathrm{N}}(=0$ for aPMA and 0.25 for iPMA) conditions, where multimolecular aggregates are present in solution in equilibrium with individual coils. Afterwards, the case of $\alpha_{\mathrm{N}}=1$ is investigated, where intermolecular association is absent, but the so-called polyelectrolyte mode sets in (for details on the discrimination between aggregation and slow mode, see the discussion in the following section). The polymer concentration range for the studies of concentration dependence was 1-3 $\mathrm{g} \mathrm{L}^{-1}$, which is below the overlap concentration, $c_{\mathrm{p}}{ }^{*}$, in all cases (as estimated by DLS, the smallest $c_{\mathrm{p}}{ }^{*}$ in our studies was $\approx 40 \mathrm{~g} \mathrm{~L}^{-1}$ ). These studies were conducted only in $0.1 \mathrm{M} \mathrm{NaCl}$.

Low $\alpha_{\mathbf{N}}$. The diffusion coefficients of individual chains, $D_{1}$, at $\alpha_{\mathrm{N}}=0$ (aPMA) and 0.25 (iPMA) were independent of $c_{\mathrm{p}}$ (Fig. 2a; the data in $0.1 \mathrm{M} \mathrm{LiCl}$ and $\mathrm{CsCl}$ at $c_{\mathrm{p}}=2 \mathrm{~g} \mathrm{~L}^{-1}$ are added for comparison), implying that the value of the second virial coefficient, $A_{2}$, for both PMAs in $0.1 \mathrm{M} \mathrm{NaCl}$ at $25{ }^{\circ} \mathrm{C}$ is close to 0 .
This is in agreement with the literature data for aPMA in aqueous $\mathrm{NaCl}^{12,38,40}$ Consequently, $R_{\mathrm{h}, 1}$ values measured at finite concentrations are close to those extrapolated to $c_{\mathrm{p}}=$ 0 and may be mutually compared. The $R_{\mathrm{h}, 1}$ of aPMA $\left(\alpha_{\mathrm{N}}=0\right)$ was from 11 to $13 \mathrm{~nm}$ and that of iPMA $\left(\alpha_{\mathrm{N}}=0.25\right)$ was somewhat smaller, from 8 to $10 \mathrm{~nm}$, irrespective of the nature of the cation.

The small $R_{\mathrm{h}, 1}$ values of individual chains are expected, knowing that PMA chains adopt a coiled conformation for $\alpha_{\mathrm{N}}<$ $\sim 0.3$. The size of an ideal coil of a polymer chain with the same degree of polymerization as aPMA (DP $\approx 2200)$ or iPMA (DP $\approx$ 370 ) would have to be around 10 and $4 \mathrm{~nm}$, respectively, which suggests that the determined size of iPMA is too large. Likely reasons for this are more chain rigidity of the stereoregular iPMA and its non-zero $\alpha_{\mathrm{N}}$ value.

In contrast to $R_{\mathrm{h}, 1}, c_{\mathrm{p}}$ has a significant effect on the hydrodynamic radius of the aggregates, $R_{\mathrm{h}, 2}$. As expected, the extent of aggregation, as the size of the aggregates, increases with increasing $c_{\mathrm{p}}$ from 90 to $122 \mathrm{~nm}$ ( 250 to $340 \mathrm{~nm}$ ) at $c_{\mathrm{p}}=1.5$ and $2.5 \mathrm{~g} \mathrm{~L}^{-1}$ for iPMA and aPMA, respectively. However, the shape parameter $\rho\left(=R_{\mathrm{g}, 2} / R_{\mathrm{h}, 2}\right)$, which will be discussed in detail below, is independent of $c_{\mathrm{p}}(\rho \approx 0.60$ and 0.73 for iPMA and aPMA, respectively), showing that the aggregates remain selfsimilar. On the basis of this, we propose that the interpretation of aggregation phenomena in dilute PMA solutions at low $\alpha_{\mathrm{N}}$ is not affected by the polymer concentration as long as solutions are sufficiently dilute (well below $c_{\mathrm{p}}{ }^{*}$ ).

High $\alpha_{\mathrm{N}}$. At higher $\alpha_{\mathrm{N}}$, however, charge interactions between polyions strongly affect their dynamic behaviour in solution. In addition to the fast diffusion, a considerably slower diffusive process (in the Introduction highlighted as the slow mode) appears due to the electrostatic repulsion between polyions. It has been shown by Förster et al..$^{27,28}$ that the prime parameter in the appearance of the slow mode is the ratio of the polyion charge, $c_{\mathrm{pc}}$, to the added salt concentration, $c_{\mathrm{s}}$, denoted by the authors as parameter $\lambda\left(=c_{\mathrm{pc}} / c_{\mathrm{s}}\right)$, and not the absolute polymer or salt concentrations. Three regimes were identified in 


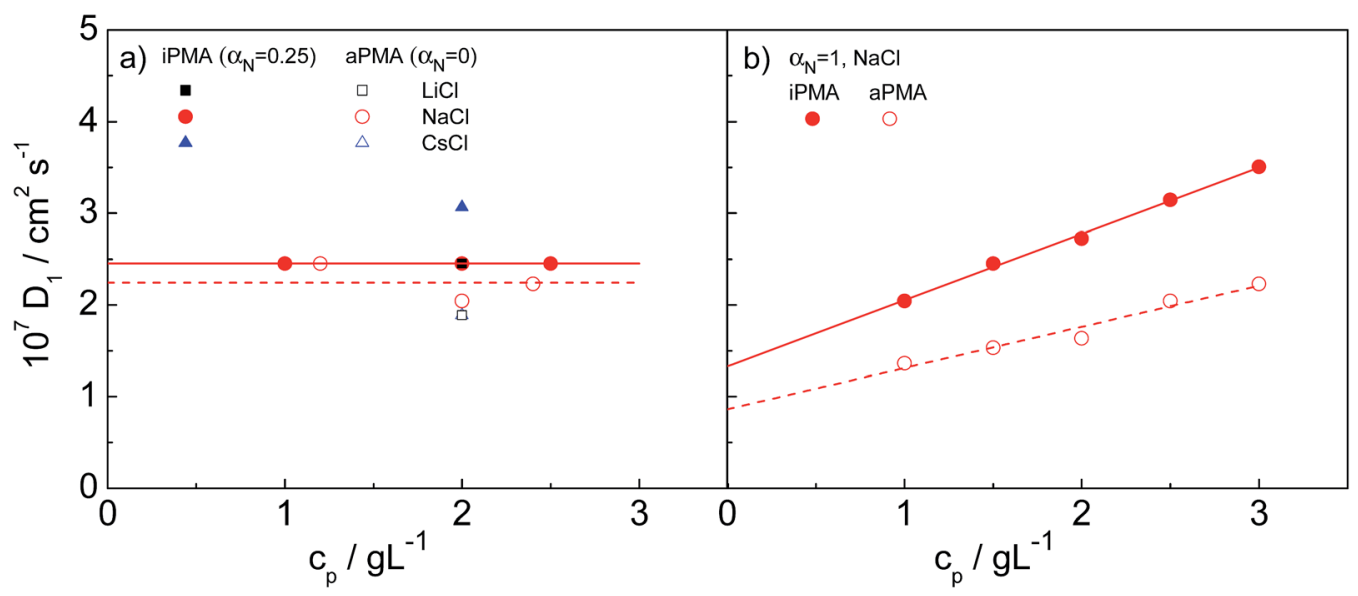

Fig. 2 The dependence of the diffusion coefficient of individual chains, $D_{1}$, on polymer concentration, $C_{p}$, in $0.1 \mathrm{M} \mathrm{NaCl}$ (a) iPMA at $\alpha_{N}=0.25$ and aPMA at $\alpha_{\mathrm{N}}=0$ (for comparison, values in $0.1 \mathrm{M} \mathrm{LiCl}$ and $\mathrm{CsCl}$ at $c_{\mathrm{p}}=2 \mathrm{~g} \mathrm{~L}^{-1}$ are also shown) and (b) both PMAs at $\alpha_{\mathrm{N}}=1$ in $0.1 \mathrm{M} \mathrm{NaCl}$. Lines in Fig. 2a only apply to the data in $0.1 \mathrm{M} \mathrm{NaCl}$.

polyelectrolyte solutions in relation to the value of $\lambda$ : (i) a "dilute lattice" regime ( $\lambda \ll 1$; excess of salt) with a single diffusion coefficient related to coupled diffusion of individual polyions and bound counterions, (ii) a transition regime $(\lambda \approx 1)$, where the slow mode gradually appears, and (iii) a "semidilute lattice" regime $(\lambda \gg 1$; excess of polyion charge), where electrostatic forces between polyions lead to formation of a transient network (sometimes called multimacroion domains), ${ }^{30}$ which is detected in LS measurements as this slow mode.

In our study, $c_{\mathrm{s}}$ was constant $\left(c_{\mathrm{s}}=0.1 \mathrm{M}\right)$ and $\lambda$ was varied via $\alpha_{\mathrm{N}}$, which affects $c_{\mathrm{pc}}\left(c_{\mathrm{pc}}=\alpha_{\mathrm{N}} c_{\mathrm{p}}\right)$. The maximum value of $\lambda$ was 0.235 for fully ionized polyions $\left(\alpha_{N}=1\right)$, meaning that the conditions in PMA solutions correspond to the transient or possibly the "dilute lattice" regime. The $D_{1}$ values for $\alpha_{\mathrm{N}}=1$ in $0.1 \mathrm{M} \mathrm{NaCl}$ are plotted in dependence on $c_{\mathrm{p}}$ in Fig. $2 \mathrm{~b}$ and are comparable in magnitude to those of weakly charged PMA chains at $c_{\mathrm{s}}=0.1 \mathrm{M}$ (compare Fig. 2a and b). However, in contrast to the low $\alpha_{\mathrm{N}}$ conditions, $D_{1}$ values increase with increasing $c_{\mathrm{p}}$, pointing to a positive value of $A_{2}$ for both PMAs at $\alpha_{\mathrm{N}}=1$, which is expected for fully charged polyions dissolved in an aqueous medium. The calculated $R_{\mathrm{h}, 1}$ values of aPMA (iPMA) chains decrease from around 18 (12) nm at $c_{\mathrm{p}}=1 \mathrm{~g} \mathrm{~L}^{-1}$ to around $11(7) \mathrm{nm}$ at $c_{\mathrm{p}}=3 \mathrm{~g} \mathrm{~L}^{-1}$.

Further experiments were performed at a constant polymer concentration of $c_{\mathrm{p}}=2 \mathrm{~g} \mathrm{~L}^{-1}$. As will become evident below, the slow diffusion process (slow mode) in PMA solutions in this polyion concentration regime depends primarily on the polyion charge, its tacticity and the type of the added cation.

\section{Aggregation at $\alpha_{\mathrm{N}}=0$ (aPMA) and 0.25 (iPMA) and the nature of aggregates}

The presence of aggregates in both iPMA and aPMA solutions at low $\alpha_{\mathrm{N}}$ is clearly manifested in the plot of the LS intensity in absolute units, $\Delta R_{\theta=0}\left(=\left(R-R_{0}\right)_{\theta=0}\right.$, where $R$ is the Rayleigh ratio of the solution and $R_{0}$ that of the solvent and the index " $\theta$ $=0$ " denotes extrapolation to zero angle) as a function of $\alpha_{\mathrm{N}}$, shown in Fig. 3 . The $\Delta R_{\theta=0}$ values are plotted as relative values,

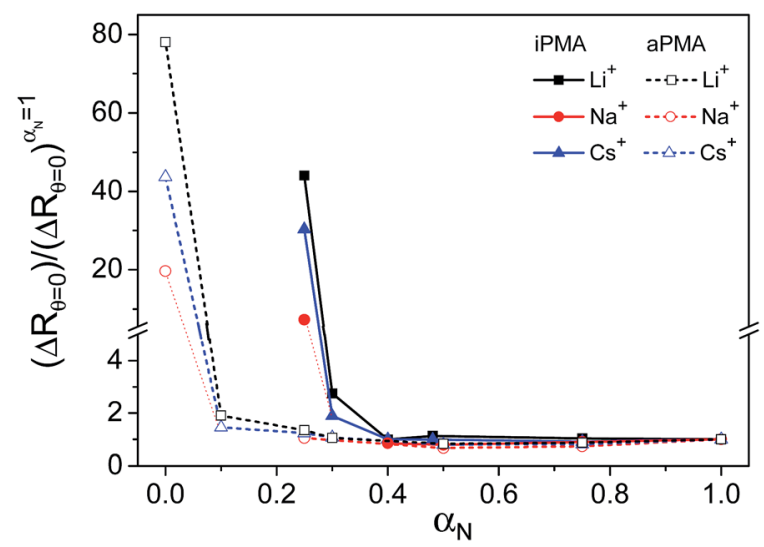

Fig. 3 The total LS intensity $\left(\Delta R_{\theta=0}\right)$ divided by its value at $\alpha_{\mathrm{N}}=$ $1\left(\left(\Delta R_{\theta=0}\right)^{\alpha_{N}=1}\right)$ plotted versus $\alpha_{N}$ in aqueous iPMA and aPMA solutions: $0.1 \mathrm{M} \mathrm{LiCl}$ (black squares), $\mathrm{NaCl}$ (red circles) and $\mathrm{CsCl}$ (blue triangles); $c_{p}=0.023 \mathrm{M}$. The solubility limit of iPMA is at $\alpha_{N} \approx 0.2$.

i.e. they are divided by $\left(\Delta R_{\theta=0}\right)^{\alpha_{N}=1}$, a value obtained at $\alpha_{\mathrm{N}}=1$. The calculated relaxation time, $\tau$, distribution functions are shown in Fig. 4.

Clearly, the total LS intensity is very high at $\alpha_{\mathrm{N}}=0$ for aPMA and at $\alpha_{\mathrm{N}}=0.25$ for iPMA and drops by 1-2 orders of magnitude at higher $\alpha_{\mathrm{N}}$. The calculated distributions at the lowest $\alpha_{\mathrm{N}}$ are bimodal in both cases. The fast relaxation time, resulting in a smaller size, $R_{\mathrm{h}, 1}$, is associated with individual chains, whereas the slow relaxation process, resulting in a larger size, $R_{\mathrm{h}, 2}$, is attributed to multi-chain aggregates. The evidence that the slow relaxation process is due to aggregates is given by the high LS intensity in these $\alpha_{\mathrm{N}}$ conditions. Data in Fig. 3 demonstrate that the intensity drops sharply with increasing $\alpha_{\mathrm{N}}$. Thus, the ratio $\Delta R_{\theta=0} /\left(\Delta R_{\theta=0}\right)^{\alpha_{N}=1}$ is close to 1 already at $\alpha_{\mathrm{N}}=0.1(0.3)$ for aPMA (iPMA), suggesting the disintegration of the aggregates in a very narrow region of $\alpha_{\mathrm{N}}$ values (the same conclusions are also obtained by considering separately the contribution of the aggregates to the total LS intensity). We conclude that the aggregation ceases abruptly with increasing $\alpha_{\mathrm{N}}$ for both PMAs. 

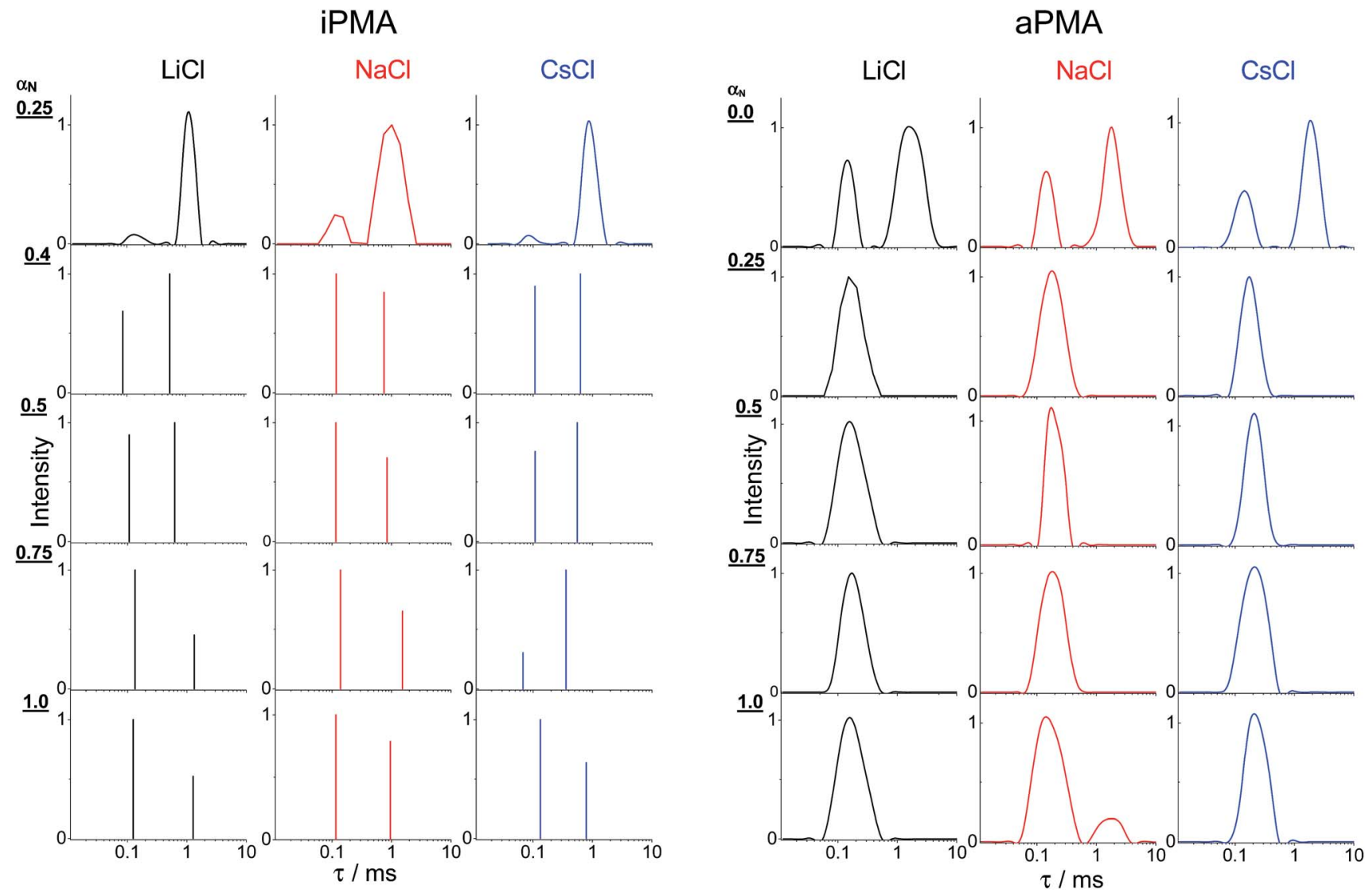

Fig. 4 The relaxation time, $\tau$, distribution functions obtained at $\theta=90^{\circ}$ for aqueous aPMA and iPMA solutions of various $\alpha_{N}$ values in $0.1 \mathrm{M}$ LiCl (black), $0.1 \mathrm{M} \mathrm{NaCl}$ (red) and $0.1 \mathrm{M} \mathrm{CsCl}$ (blue). Note that the distributions for all iPMA solutions with $\alpha_{\mathrm{N}} \geq 0.4$ were estimated using a biexponential fit (Origin 8.0 Software), whereas the others were obtained using the CONTIN program (see ESI $\dagger$ ).

The $R_{\mathrm{h}, 2}$ values of aggregates were obtained from the measured diffusion coefficients extrapolated to zero angle (see Fig. S5a†) and are reported in Table 2. They clearly depend on the type of the cation. $R_{\mathrm{h}, 2}$ ranges from 90 to $115 \mathrm{~nm}$ for iPMA and from 230 to $290 \mathrm{~nm}$ for aPMA and points to extensive aggregation between chains. In view of the fact that aggregates are large in size in comparison to the wavelength of light, the radius of gyration, $R_{\mathrm{g}, 2}$, could be determined from the angular dependence of the LS intensity (see Fig. S5b $\dagger$ ), and from that the shape parameter $\rho\left(=R_{\mathrm{g}, 2} / R_{\mathrm{h}, 2}\right)$ was calculated. The resulting $\rho$ values are around 0.63 for iPMA and around 0.75 for aPMA (Table 2), which are both lower than $\rho$ for a hard sphere $(\rho=0.78)$.
In view of these small $\rho$ values, we tried to classify the aggregate structure by comparing the scattering data with theoretically predicted scattering functions for some typical particle topologies. ${ }^{39}$ For this purpose, the angular dependence of scattered light was followed and the intensities were split into contributions of small and large particles as demonstrated in ESI. $\dagger$ The data for the large particles (aggregates) are presented in Fig. 5 in the form of a Kratky plot (i.e. the dependence of $\left(q R_{\mathrm{g}}\right)^{2} P(q)$ on $\left.q R_{\mathrm{g}}\right)$. The experimental points are compared with six selected particle topologies, ranging from rigid rod to hard sphere. It can be seen that the majority of data points for iPMA remain in the range of low $q$-values (corresponding to $q R_{\mathrm{g}}<1.5$ ) where most of the topologies (except the spherical) fit. The aggregates of aPMA are sufficiently large to exceed this limit.

Table $2 R_{\mathrm{h}, 1}, R_{\mathrm{h}, 2}, R_{\mathrm{g}, 2}$ (all in $\mathrm{nm}$ ) and the shape parameter $\rho\left(=R_{\mathrm{g}, 2} / R_{\mathrm{h}, 2}\right)$ obtained from SLS and DLS measurements in $0.1 \mathrm{M} X C l$ solutions of iPMA and aPMA $\left(c_{\mathrm{p}}=0.023 \mathrm{M}\right)$ at $25^{\circ} \mathrm{C}: \alpha_{\mathrm{N}} \approx 0.25$ (iPMA) and $\alpha_{\mathrm{N}}=0$ (aPMA)

\begin{tabular}{|c|c|c|c|c|c|c|c|c|c|c|}
\hline \multirow[b]{2}{*}{$\mathrm{XCl}$} & \multicolumn{5}{|c|}{ iPMA } & \multicolumn{5}{|c|}{ aPMA } \\
\hline & $\alpha_{\mathrm{N}}$ & $R_{\mathrm{h}, 1}$ & $R_{\mathrm{h}, 2}$ & $R_{\mathrm{g}, 2}$ & $\rho$ & $\alpha_{\mathrm{N}}$ & $R_{\mathrm{h}, 1}$ & $R_{\mathrm{h}, 2}$ & $R_{\mathrm{g}, 2}$ & $\rho$ \\
\hline $\mathrm{NaCl}$ & 0.25 & $10 \pm 1$ & $110 \pm 2$ & $64 \pm 2$ & $0.58 \pm 0.02$ & 0 & $12 \pm 1$ & $292 \pm 5$ & $221 \pm 3$ & $0.76 \pm 0.02$ \\
\hline $\mathrm{CsCl}$ & 0.27 & $7 \pm 1$ & $90 \pm 2$ & $60 \pm 2$ & $0.67 \pm 0.03$ & 0 & $13 \pm 1$ & $231 \pm 4$ & $173 \pm 3$ & $0.75 \pm 0.02$ \\
\hline
\end{tabular}




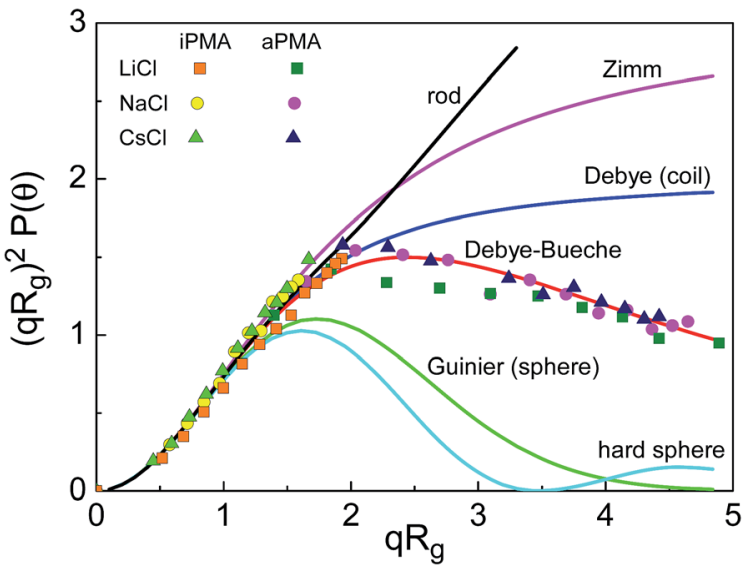

Fig. 5 The Kratky plot for six selected topologies (solid lines; for details see ref. 39) and the experimental data for $\operatorname{iPMA}(\square, 0, \mathbf{\Delta})$ and aPMA $(\square, 0$, $\Delta)$ at $\alpha_{N}=0.25$ and 0 , respectively, in $0.1 \mathrm{M} \mathrm{LiCl}$ (squares), $\mathrm{NaCl}$ (circles), and $\mathrm{CsCl}$ (triangles). The LS data in this figure apply to the slow diffusive process, thus only representing the multimolecular aggregates.

Thus the corresponding data extend to higher $q R_{\mathrm{g}}$ values and can clearly be approximated with the Debye-Bueche scattering function (for details of these fits, see also Fig. S5b $†$ ). As is seen in Fig. 5, the iPMA aggregates are too small to resolve their internal structure using the Kratky plot. However, it appears that the calculated points correspond to the aPMA series.

The Debye-Bueche scattering function is reported in the literature for the description of hyperbranched flexible chain molecules and microgels from chemically cross-linked flexible chains. ${ }^{41,42}$ On the basis of this, and of the low $\rho$ values, we propose that IPMA and aPMA aggregates can be characterized as microgel-like particles, i.e. spherical solvent-draining particles with a higher polymer density in the centre and a lower density towards the outer surface (shell). The "cross-links" connecting the PMA chains are physical rather than chemical.

According to the higher $\rho$ value (around 0.75 , irrespective of the added cation), the aggregates of aPMA chains have a looser structure that allows easier penetration of solvent into the interior, whereas aggregates of a highly regular iPMA with a lower $\rho$ are considerably denser with a more compact core, allowing only the outer shell to be drained by water. In addition, the $\mathrm{d} n / \mathrm{d} c_{\mathrm{p}}$ data indicate that the surface of the aggregates (providing the optical contrast in scattering) is also different for iPMA aggregates in comparison with aPMA aggregates. This may partly arise from the different extent of ion binding, but predominantly it is attributed to the different polarity of the aggregate surface.

The idea of different compactness and surface polarity of the iPMA aggregates becomes plausible if one takes into account that the iPMA chains adopt a partly helical conformation in solution. ${ }^{5,6,43}$ Such an ordered conformation would promote association through very efficient hydrogen bonding between helices arranged in parallel. It was proposed by van den Bosch et al. ${ }^{6}$ that hydrogen bonding in iPMA solutions may be strongly cooperative. For the cooperativity to be truly effective, the distribution of dissociated and undissociated carboxyl groups should not be random, as usually thought. On the contrary, we propose that the undissociated carboxyl groups $(\mathrm{COOH})$ are concentrated in the helical regions and the dissociated $\left(\mathrm{COO}^{-}\right)$ groups in the disordered regions of the chains. In this way, it becomes reasonable to suggest that domains are formed, within which uncharged portions of iPMA chains are strongly hydrogen-bonded to neighbouring chains in a cooperative manner. Water may be largely excluded from these regions (the cores of the particles). The water-exposed regions of the aggregate surface are made up of less ordered chain portions with hydrophilic $\mathrm{COO}^{-}$groups and comprise the shell around the core. The comparison of results for aPMA and iPMA implies that the affinity of charged/polar groups for water (in our case either $\mathrm{COO}^{-}$or $\mathrm{COOH}$ ) depends considerably on the particular distribution of other hydrophilic and hydrophobic groups in a certain chain conformation. The hydration/dehydration effects are thus crucial in aggregation between PMA chains, as discussed recently also by Burrows et $a l^{44}$ for another anionic polyelectrolyte, poly(vinyl sulfate), in that case with trivalent counterions. There exists only a narrow window of $\alpha_{\mathrm{N}}$ in which the described association in PMA solutions can be observed.

A sketch of the proposed model for the aggregation of iPMA chains is presented in Fig. 6 . The first step in this model is the disorder-to-order (coil-to-helix) transition and the second one is the side-by-side aggregation of helices. A similar cooperative mechanism was reported previously for intermolecular association between even more hydrophobic poly(ethacrylic acid), PEA, chains in water ${ }^{45}$ and for synthetic polymers in organic solvents, e.g. for isotactic poly(methylmethacrylate) in 2-butanone $^{46}$ or syndiotactic poly(styrene) in bromoform. ${ }^{47}$ The majority of the ionized carboxyl groups in this aggregate are exposed to the solvent and the hydrophobic methyl groups are mostly hidden in the hydrophobic core. Thus the dielectric constant of the hydrophobic core is lower than that of the surrounding shell, as proved by fluorescence measurements. ${ }^{5}$ The charges on the iPMA chains together with their counterions only exist in the shell of the aggregates, which are therefore swollen with water due to the osmotic pressure effects.

On the other hand, aPMA chains have a random distribution of carboxyl groups along the chain and at $\alpha_{\mathrm{N}}=0$ only a very small part (a few \%) is charged due to self-ionization. The $\mathrm{COOH}$ groups are capable of forming intermolecular hydrogen bonds, but presumably in a less cooperative manner due to aPMA's irregular structure. Consequently, the core of aPMA

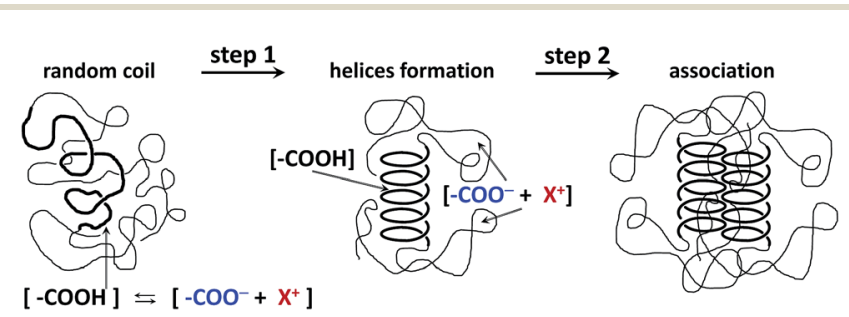

Fig. 6 A model of the association process in iPMA solutions at $\alpha_{N}=$ 0.25 . 
aggregates is less dense in comparison with those of iPMA and at the same time the surface has a considerably lower charge. Besides, it was demonstrated previously by pyrene-based fluorescence measurements that the interior of the compact form of aPMA at $\alpha_{\mathrm{N}}=0$ is actually more hydrophobic than that of iPMA, because the methyl groups can be more efficiently buried in it, ${ }^{5}$ whereas the surface has to be more hydrophilic. This is finally demonstrated also in a different optical contrast. Responsibility for the stability of aPMA aggregates in solutions at rest comes largely from the hydrophobic effect associated with the methyl side groups, and not so much from hydrogen bonding, as is the case with iPMA. However, we have to stress that the probability of hydrogen bond formation between aPMA chains strongly increases in the field of flow as confirmed in several publications, ${ }^{9,11,13}$ which is not the case with iPMA. ${ }^{13}$

Generally, aggregation is more favourable for neutral polymers and increases with increasing $M_{\mathrm{w}}$. Thus our results suggest that iPMA, which is ionized to a non-negligible degree $\left(\alpha_{\mathrm{N}}=0.25\right)$ and has a six times lower $M_{\mathrm{W}}$ than aPMA, is much more inclined towards intermolecular association than aPMA.

\section{Effect of ions on aggregation}

The sequence of ions in relation to the size of the aggregates is $\mathrm{Li}^{+}>\mathrm{Na}^{+}>\mathrm{Cs}^{+}$for iPMA and $\mathrm{Na}^{+}>\mathrm{Li}^{+}>\mathrm{Cs}^{+}$for aPMA (see Table 2 ), the same as noticed previously for $\mathrm{d} n / \mathrm{d} c_{\mathrm{p}}$ values. These results show that $\mathrm{Na}^{+}$and $\mathrm{Li}^{+}$better support aggregation between iPMA and aPMA chains than $\mathrm{Cs}^{+}$ions do. This observation can be understood by taking into account the hydration radii of the investigated cations, which decrease with increasing ionic radii, and by applying the LMWA ${ }^{17-19}$ for the interpretation of ion pair formation in the electrolyte solutions. The $\mathrm{COO}^{-}$ anion is strongly hydrated, as are the $\mathrm{Li}^{+}$and $\mathrm{Na}^{+}$ions; they have high hydration enthalpies, $\Delta H_{\text {hydr }}$. On the other hand, the $\mathrm{Cs}^{+}$ ion is only weakly hydrated, with a considerably lower $\Delta H_{\text {hydr }}$. The LMWA states that the closer the $\Delta H_{\text {hydr }}$ values of ions are, the stronger is the resulting ion pair. Thus $\mathrm{COO}^{-}$is expected to form the strongest ion pair with $\mathrm{Na}^{+}$ions, since their $\Delta H_{\text {hydr }}$ values are very close to each other $\left(\Delta H_{\text {hydr }} \approx-420 \mathrm{~kJ} \mathrm{~mol}^{-1}\right)$, and the weakest one with $\mathrm{Cs}^{+}$ions.

Although PMA chains at low $\alpha_{\mathrm{N}}$ are only weakly charged (a few $\mathrm{COO}^{-}$groups are present due to self-ionization in the aPMA $\alpha_{\mathrm{N}}=0$ case, and around $25 \%$ in the iPMA $\alpha_{\mathrm{N}} \approx 0.25$ case), the LMWA seems to apply fairly well. Thus $\mathrm{Na}^{+}$ions better support aggregation between PMA chains than $\mathrm{Cs}^{+}$ions do, so larger aggregates are formed in the former case. The observed sequence in the size of aPMA aggregates $\left(\mathrm{Na}^{+}>\mathrm{Li}^{+}>\mathrm{Cs}^{+}\right)$is therefore in perfect agreement with the LMWA, although aPMA is virtually uncharged. Recent computer simulations by Hess et $a{ }^{48}$ performed on a neutral protein and on two (either positively or negatively) charged proteins show that the neutral protein followed the same series as did the charged proteins with respect to ion specificity. The authors have concluded that this is probably due to the fact that carboxylates stick out of the protein surface and are fully hydrated. This is also a reasonable explanation for aPMA. On the level of an individual chain, it is widely accepted that aPMA at low $\alpha_{\mathrm{N}}$ is in a rather compact coil conformation. Methyl groups are predominantly buried in the core of such a structure (the core is rather hydrophobic; see above) and polar $\mathrm{COOH}$ groups, and among these some charged $\mathrm{COO}^{-}$groups arising from self-ionization, are facing the water solvent.

In the iPMA case, the largest aggregates are formed in the presence of $\mathrm{LiCl}$, suggesting that $\mathrm{Li}^{+}$ions interact more strongly with carboxylates on the iPMA chain. Several factors may be responsible for this difference in comparison with aPMA. First, it is reasonable to expect that the $\Delta H_{\text {hydr }}$ of an ion depends to some extent also on the structural features of the whole macromolecule. The particular positioning of the $\mathrm{COO}^{-}$groups on the isotactic chain could lead to a modified interaction of the aggregated isotactic polymer with water. Water molecules avoid contact with hydrophobic methyl groups and are pushed toward ionized $\mathrm{COO}^{-}$groups with a high affinity for water. In this way, they may contribute to a somewhat higher $\Delta H_{\text {hydr }}$ of $\mathrm{COO}^{-}$, closer to that of $\mathrm{Li}^{+}$(for which $\Delta H_{\mathrm{hydr}}=-531 \mathrm{~kJ} \mathrm{~mol}^{-1}$ ). Besides, it is also known that the $\mathrm{Li}^{+}$ion's position in the Hofmeister series shifts depending on the surrounding environment. These together could bring about a different sequence in iPMA solutions in comparison with those of aPMA. An important implication of our results is that the cation specificity in PMA solutions is determined by the properties of the cation's water of hydration and by the ability to donate these water molecules to the polymer, which certainly depends on the polymer's state in solution.

It is worth noting that the value of $\rho$ (see Table 2 ) shows no pronounced ion-specific effects. The same also holds for the Kratky plot (Fig. 5). This implies that the nature (spatial mass distribution) of the aggregates is similar, irrespective of the added ion, but only the extent of aggregation changes. The mass distribution within the aggregates is affected primarily by the chemical and physical nature of the polyion.

\section{The $\alpha_{\mathrm{N}}$ dependence of the slow mode}

We now turn our attention to the study of the slow mode as a function of $\alpha_{\mathrm{N}}$. Fig. 3 shows that the total LS intensity in the presence of all XCls drops to a very low and constant value for $\alpha_{\mathrm{N}}$ $>0$ (aPMA) or $\alpha_{\mathrm{N}}>0.25$ (iPMA). All time distribution functions for iPMA solutions with $\alpha_{\mathrm{N}}>0.25$ are bimodal, regardless of the counterion (Fig. 4). As for aPMA, bimodality is observed only for $\alpha_{\mathrm{N}}=1$ in the presence of $0.1 \mathrm{M} \mathrm{NaCl}$. The fast mode, as before, is associated with the diffusion of individual chains (small particles). On the other hand, the slow mode at higher $\alpha_{\mathrm{N}}$ does not represent particles, but is due to the so-called polyelectrolyte effect, originating from electrostatic repulsions between the charged chains. The evidence for this is provided by the low LS intensity (c.f. Fig. 3) and by inspecting plots of the decay rate, $\Gamma$, in dependence on the square of the scattering vector $q$ given in Fig. 7. An additional verification that we are dealing with an "anomalous" diffusion process is that points are rather scattered in the Kratky plot (not reported); it is impossible to claim that they follow any specific topology.

Curves with a high slope in Fig. 7 correspond to the fast relaxation process, characterized by the fast relaxation rate, $\Gamma_{\mathrm{f}}$, 


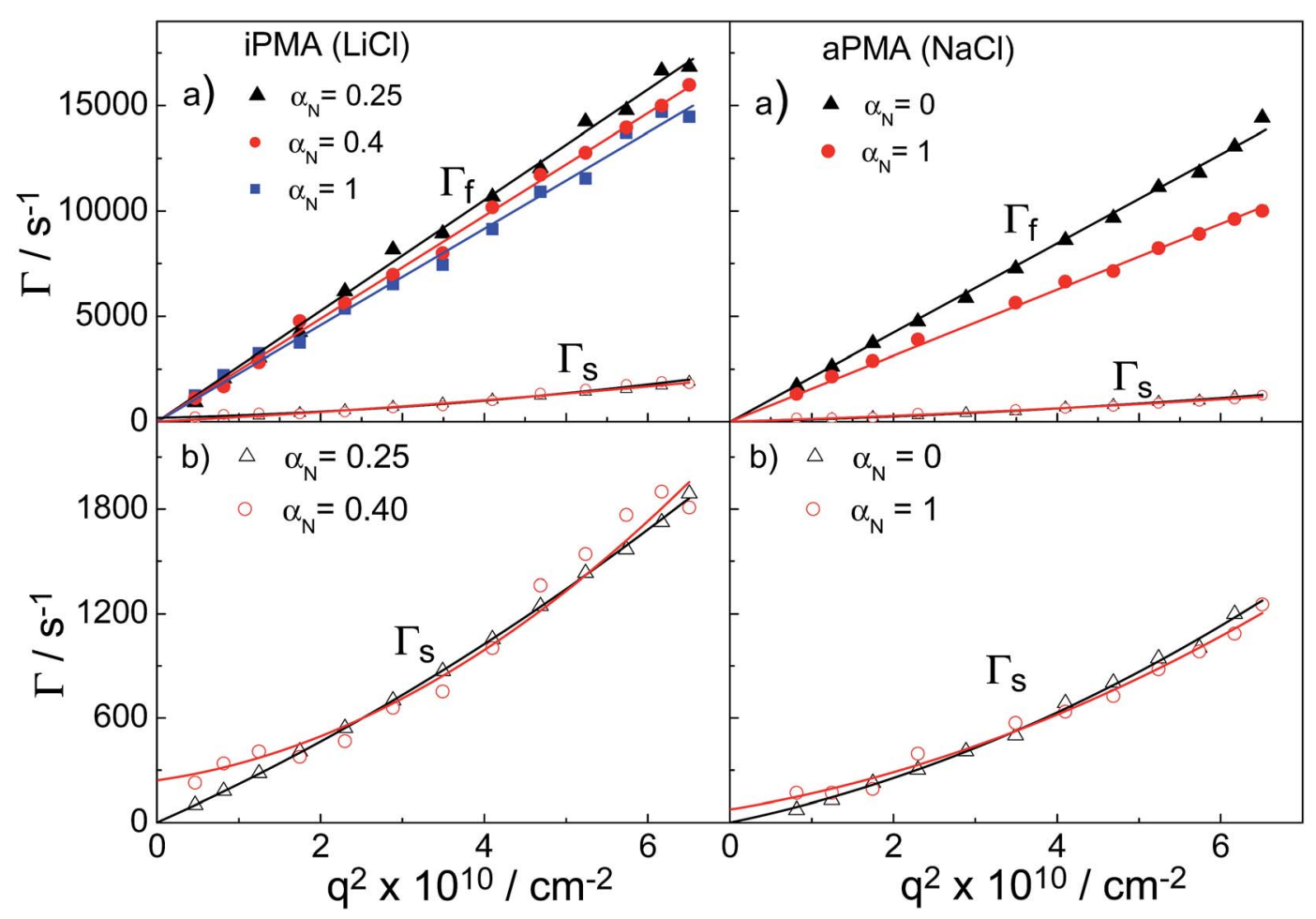

Fig. 7 Decay rates, $\Gamma=\tau^{-1}$, as a function of the square of the scattering vector, $q^{2}$, at selected $\alpha_{N}$ values for iPMA in $0.1 \mathrm{M} \mathrm{LiCl}$ and for aPMA in 0.1 $\mathrm{M} \mathrm{NaCl}$ : (a) fast, $\Gamma_{\mathrm{f}}$ (filled symbols), and slow mode, $\Gamma_{\mathrm{s}}$ (open symbols); (b) only slow mode, $\Gamma_{\mathrm{s}}$.

and those with the low slope to a considerably slower relaxation process, characterized by the slow relaxation rate, $\Gamma_{\mathrm{s}}$. In Fig. $7 \mathrm{~b}$, the range for $\Gamma_{\mathrm{s}}$ curves is enlarged. At all studied $\alpha_{\mathrm{N}}$ values and for all XCls, the $\Gamma_{\mathrm{f}} v s . q^{2}$ curves were linear for both PMAs. They start from the origin, thus representing true translational diffusion of rather monodisperse (and small) particles. Also, the $\Gamma_{\mathrm{s}}$ vs. $q^{2}$ curves for $\alpha_{\mathrm{N}}=0$ (aPMA) or 0.25 (iPMA) start from the origin, but show positive deviations from linearity with increasing $q^{2}$ as a consequence of polydispersity of the aggregates.

In contrast, the $\Gamma_{\mathrm{s}}$ curves at higher $\alpha_{\mathrm{N}}$ do not go through the origin (c.f. the lines in Fig. 7b for $\alpha_{\mathrm{N}}=0.4$ (iPMA) and 1 (aPMA) and additional curves for iPMA with $\alpha_{\mathrm{N}}=1$ in ESI, Fig. S6†) and cannot be related to the translational diffusion. ${ }^{34}$ They are due to the polyelectrolyte effect, often referred to in the literature as the slow mode. ${ }^{25-28}$ Note that we use the notation $\Gamma_{\mathrm{s}}$ for both the aggregates and the slow mode, because it signifies the slow diffusion (real or "anomalous") characterized by a small value of $D$. Similar curves were reported in Cong et al..$^{25}$ (c.f. Fig. 2 there) for sodium poly(styrenesulfonate), NaPSS, where the slow mode was studied in detail. Our results show that the slow mode is present in the aqueous $0.1 \mathrm{M}$ XCls solutions of iPMA at all $\alpha_{\mathrm{N}} \geq$ 0.4 , whereas for aPMA it is only detected in the $0.1 \mathrm{M} \mathrm{NaCl}$ solution at $\alpha_{\mathrm{N}}=1$. This indicates that under the investigated conditions, $0.1 \mathrm{M}$ alkali chlorides do not provide efficient screening of the electrostatic forces between iPMA chains, whereas they do for aPMA chains in all the cases except for the highest polyion charge $\left(\alpha_{\mathrm{N}}=1\right)$ in $0.1 \mathrm{M} \mathrm{NaCl}$. This difference between aPMA and iPMA agrees with the conclusions of previous investigations ${ }^{5,7}$ that the iPMA chain has a locally higher charge density in comparison to aPMA and therefore a higher salt concentration is needed to screen electrostatic interactions efficiently. The $0.1 \mathrm{M}$ XCls seem to be the borderline case for observation of the slow mode, at least as far as aPMA is concerned. Further investigations are in progress regarding the dependence of the slow mode in IPMA and aPMA solutions on salt concentration. One of the open issues is also the effect of ions on the slow mode.

The diffusion coefficients were obtained from the initial slopes of the decay rate curves and are plotted in Fig. 8 as a function of $\lambda$. The range of $\lambda$ values that can be covered by changing $\alpha_{\mathrm{N}}$ (at constant $c_{\mathrm{p}}$ and $\left.c_{\mathrm{s}}\right)$ is rather narrow $(0.01<\lambda<$ 0.24). In this $\lambda$ range, the $D_{\mathrm{f}}$ of the iPMA solutions, as of the aPMA solutions (for $\lambda>0.05$ or $\alpha_{\mathrm{N}}>0.2$ ) is more or less independent of $\alpha_{\mathrm{N}}$ in all $0.1 \mathrm{M}$ XCls. Somewhat higher values are obtained only for aPMA at $\alpha_{\mathrm{N}}=0$ (practically uncharged chains) for all three salts. The latter is related to the faster diffusion of the uncharged chains in comparison to the charged chains at the rather high ionic strength provided by $0.1 \mathrm{M}$ XCls. In contrast to our result, Sedlak ${ }^{26,31}$ has reported an increasing trend of $D_{\mathrm{f}}$ with $\alpha_{\mathrm{N}}(\lambda)$ up to $\alpha_{\mathrm{N}} \approx 0.5(\lambda \approx 0.12)$, however at a considerably higher $c_{\mathrm{p}}\left(=36.6 \mathrm{~g} \mathrm{~L}^{-1}\right)$ and in salt-free aPMA solutions. Förster et al. ${ }^{27}$ report diffusion coefficients of quaternized poly(2-vinyl pyridine), QPVP, over a much broader range of $\lambda$ values (from around 0.005 to more than 1000). Similarly to our case, no strong dependence of $D_{\mathrm{f}}$ on $\lambda$ can be inferred for QPVP in the same $\lambda$ range as covered in our work $(0.01<\lambda<0.24)$. The slow diffusion coefficient, $D_{\mathrm{s}}$, of PMA 


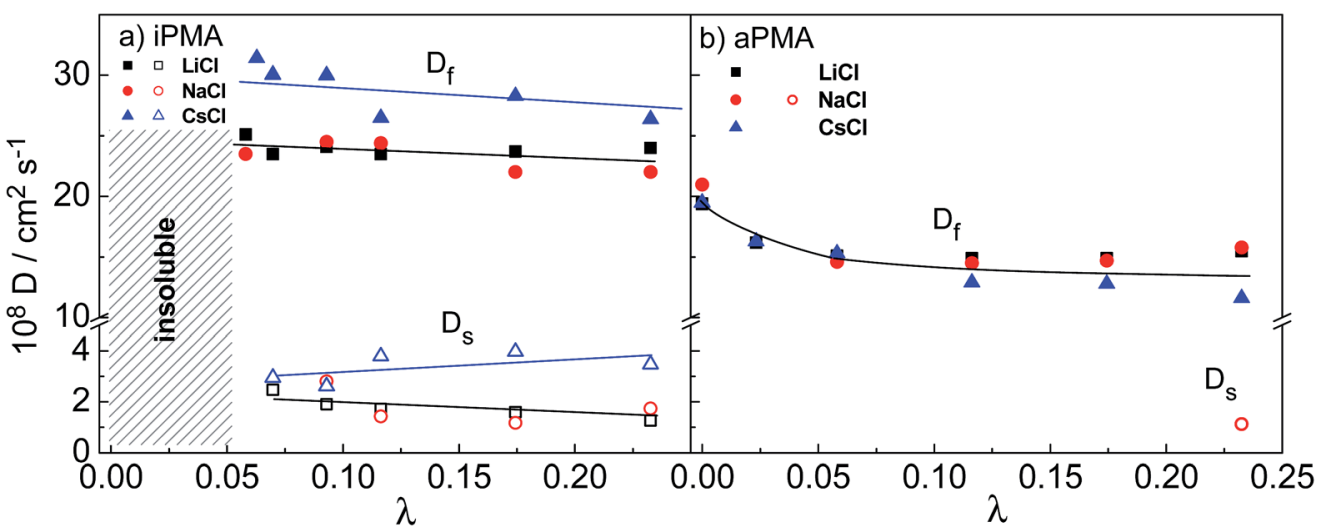

Fig. 8 Fast, $D_{f}$, and slow, $D_{s}$ diffusion coefficients as a function of the parameter $\lambda\left(=\alpha_{N} C_{p} / C_{s}\right)$ for (a) iPMA and (b) aPMA in the presence of $0.1 \mathrm{M}$ $\mathrm{LiCl}$ (black squares), $\mathrm{NaCl}$ (red circles) and $\mathrm{CsCl}$ (blue triangles). The filled symbols denote the fast relaxation mode and the open symbols the slow relaxation mode.

differs from $D_{\mathrm{f}}$ by approximately one order of magnitude, again similar to the QPVP case, and is almost constant in the whole $\lambda$ range. It has to be noticed that in Förster et al. ${ }^{27} \lambda$ was varied by changing $c_{\mathrm{s}}$ and $c_{\mathrm{p}}$, and not $\alpha_{\mathrm{N}}$ as in the PMA case reported here.

The effect of different alkali metal ions on $D_{\mathrm{f}}$ and $D_{\mathrm{s}}$ is not as pronounced as their effect on aggregation. It may be noticed that both the $D_{\mathrm{f}}$ and $D_{\mathrm{s}}$ of iPMA in $0.1 \mathrm{M} \mathrm{CsCl}$ are higher in comparison with $0.1 \mathrm{M} \mathrm{LiCl}$ and $\mathrm{NaCl}$ in the whole $\lambda$ region, whereas with aPMA there is no obvious difference. The explanation of these subtle differences is justified only in the case of iPMA in $0.1 \mathrm{M} \mathrm{CsCl}$ and invokes the role of cations in screening electrostatic interactions between negatively charged polyions. It is well known that polyions diffuse much faster in comparison with the equivalent neutral polymer chains due to their mutual electrostatic repulsion. If these interactions are screened, the diffusion should slow down. In the function of screening, $\mathrm{Cs}^{+}$ions are less effective than $\mathrm{Li}^{+}$and $\mathrm{Na}^{+}$ions, which stems from the effects discussed in relation to hydration/ dehydration (see above). $\mathrm{Cs}^{+}$ions are not so prone to release their water of hydration upon binding to the carboxylate groups on PMA as are $\mathrm{Li}^{+}$and $\mathrm{Na}^{+}$. They bind to the polyion's electrostatic atmosphere in accordance with the Coulomb forces. In the polyelectrolyte field, this type of binding is described as atmospheric binding in contrast to site binding, ${ }^{23}$ which involves dehydration. Therefore, $\mathrm{Cs}^{+}$ions bind less strongly to PMA than do $\mathrm{Li}^{+}$and $\mathrm{Na}^{+}$ions and thus the diffusion of polyions with carboxylate groups is faster in $\mathrm{CsCl}$ solutions. This is naturally reflected in both $D_{\mathrm{f}}$ and $D_{\mathrm{s}}$ values. As far as $\mathrm{Li}^{+}$and $\mathrm{Na}^{+}$ ions are concerned, the mutual difference in diffusion coefficients is not significant. This finding may be related to the value of $\Delta H_{\text {hydr }}$, which is closer for these two cations and considerably lower (by around 50 to $90 \%$ ) for the $\mathrm{Cs}^{+}$ions. $^{21}$

\section{Conclusions}

In conclusion, we have shown that isotactic and atactic PMA chains aggregate in aqueous solutions at low degrees of neutralization, $\alpha_{\mathrm{N}}$, in the presence of $0.1 \mathrm{M} \mathrm{LiCl}, \mathrm{NaCl}$ and $\mathrm{CsCl}$. The aggregates, irrespective of the cation present, resemble spherical microgel-like particles with a core-shell structure. The density distribution between the core and the shell depends primarily on the chain tacticity and, to a lesser extent, on the nature of the cation. The effect of the cations is revealed in the extent of aggregation. The aggregates' sizes follow the sequence (from the largest to the smallest) $\mathrm{Na}^{+}>\mathrm{Li}^{+}>\mathrm{Cs}^{+}$for aPMA and $\mathrm{Li}^{+}>\mathrm{Na}^{+}>\mathrm{Cs}^{+}$for iPMA, which was explained by the law of matching water affinities.

The iPMA chains form aggregates with a considerably denser core than the aPMA chains, as deduced from the value of the shape parameter. Not only the density distribution, but also the outer and inner polarity of the aggregates is different. Thus the aPMA aggregates have a more hydrophobic, although less compact, core. This was inferred from the measured optical contrast, as reflected by the refractive index increment values, and from previous fluorescence studies. The greater compactness of the iPMA aggregates was explained by proposing a model of cooperative hydrogen bond formation between chains in a helical conformation. The undissociated carboxyl groups are concentrated in these helical regions within the core and the dissociated groups in the disordered regions of the aggregates, i.e. in the corona. Such a model could also explain the different sequence of cations for iPMA on the one hand and for aPMA on the other hand (see above). It is suggested that the state of the hydrated iPMA aggregates is different from that of the hydrated aPMA aggregates. Consequently, the affinity of the $\mathrm{Li}^{+}$ions for the carboxylates of iPMA may become larger than that of the $\mathrm{Na}^{+}$ ions.

Different chain conformations are not limited to low $\alpha_{\mathrm{N}}$. Investigations upon increasing $\alpha_{\mathrm{N}}$ show that the slow mode, arising from the electrostatic interactions between the charged chains, is present for iPMA at all $\alpha_{\mathrm{N}}>0.25$ and in all $0.1 \mathrm{M}$ XCls, but for aPMA only at $\alpha_{\mathrm{N}}=1$ in $0.1 \mathrm{M} \mathrm{NaCl}$. The most straightforward explanation of this is that the effective charge density of both PMAs is different due to different chain conformations. The helical conformation of iPMA, which is also preserved at higher $\alpha_{\mathrm{N}}$, leads to a locally higher charge density of iPMA in comparison with aPMA. It was concluded that the $0.1 \mathrm{M}$ concentration of XCls is not sufficient to screen the electrostatic 
repulsion between iPMA chains and seems to be the borderline case for aPMA. Further investigations on the dependence of the slow mode on salt concentration in PMA solutions are in progress.

\section{Acknowledgements}

This work was financially supported by the Slovenian Research Agency, ARRS, through the Physical Chemistry program P10201. The authors are grateful to the Slovenian Research Agency, ARRS (Grants no. BI-FI/09-005, BI-FI/11-12-004), to the Academy of Finland (Grants no. 132404 and 134581), and to Finnish Society of Science and Letters (Magnus Ehrnrooth Foundation) for the financial support of the research visits.

\section{Notes and references}

1 M. Rubinstein and G. A. Papoian, Soft Matter, 2012, 8, 92659267 and papers therein.

2 V. Crescenzi, Adv. Polym. Sci., 1968, 5, 358-386.

3 K. Hatada, J. Polym. Sci., Part A: Polym. Chem., 1999, 37, 245260.

4 J. C. Leyte, H. M. R. Arbouw-van der Veen and L. H. Zuiderweg, J. Phys. Chem., 1972, 76, 2559-2561.

5 N. Vlachy, J. Dolenc, B. Jerman and K. Kogej, J. Phys. Chem. B, 2006, 110, 9061-9071.

6 E. van den Bosch, Q. Keil, G. Filipcsei, H. Berghmans and H. Raynaers, Macromolecules, 2004, 37, 9673-9675.

7 B. Jerman, M. Breznik, K. Kogej and S. Paoletti, J. Phys. Chem. $B, 2007,111,8435-8443$.

8 B. Jerman and K. Kogej, Acta Chim. Slov., 2006, 53, 264-273. 9 J. Eliassaf and A. Silberberg, Polymer, 1962, 3, 555-564.

10 J. Eliassaf, A. Silberberg and A. Katchalsky, Nature, 1955, 176, 1119.

11 S. Ohoya, S. Hashiya, K. Tsubakiyama and T. Matsuo, Polym. J., 2000, 32, 133-139.

12 K. Kogej, H. Berghmans, H. Reynaers and S. Paoletti, J. Phys. Chem. B, 2004, 108, 18164-18173.

13 S. Sitar, V. Aseyev and K. Kogej, Polymer, 2014, 55, 848-854. 14 E. M. Loebl and J. J. O'Niell, J. Polym. Sci., 1960, 45, 538-540. 15 F. Hofmeister, Arch. Exp. Pathol. Pharmakol., 1888, 24, 247260.

16 W. Kunz, J. Henle and B. W. Ninham, Curr. Opin. Colloid Interface Sci., 2004, 9, 19-37.

17 K. Collins, Biophys. J., 1997, 72, 65-76.

18 K. D. Collins, Methods, 2004, 34, 300-311.

19 K. Collins, G. W. Neilson and J. E. Enderby, Biophys. Chem., 2007, 128, 95-104.

20 W. Kunz, Curr. Opin. Colloid Interface Sci., 2010, 15, 34-39.

21 J. Kher, S. C. Flores and P. S. Cremer, J. Phys. Chem. B, 2012, 116, 7389-7397.

22 J. Heyda and J. Dzubiella, Soft Matter, 2012, 8, 9338-9344.
23 U. P. Strauss and Y. P. Leung, J. Am. Chem. Soc., 1965, 87, 1476-1480.

24 S. C. Lin, W. I. Lee and J. M. Schurr, Biopolymers, 1978, 17, 1041-1064.

25 R. Cong, E. Temyanko, P. S. Russo, N. Edwin and R. M. Uppu, Macromolecules, 2006, 39, 731-739.

26 M. Sedlak, Č. Konak, P. Štepanek and J. Jakeš, Polymer, 1987, 28, 873-879.

27 S. Förster, M. Schmidt and M. Antonietti, Polymer, 1990, 31, 781-792.

28 S. Förster and M. Schmidt, Adv. Polym. Sci., 1995, 120, 51133.

29 M. Drifford and J. P. Dalbiez, Biopolymers, 1985, 24, 15011514.

30 M. Sedlák, Macromolecules, 1993, 26, 1158-1162.

31 M. Sedlák, Langmuir, 1999, 15, 4045-4051.

32 C. Urban and P. Schurtenberger, J. Colloid Interface Sci., 1998, 207, 150-158.

33 W. Schärtl, Light Scattering from Polymer Solutions and Nanoparticle Dispersions, Springer Verlag, Berlin, Heidelberg, 2007.

34 Dynamic Light Scattering: The Method and Some Application, ed. W. Brown, Clarendon Press, Oxford, 1993.

35 E. V. Tarassova, V. Aseyev, A. Filippov and H. Tenhu, Polymer, 2007, 48, 4503-4510.

36 M. Kanao, Y. Matsuda and T. Sato, Macromolecules, 2003, 36, 2093-2102.

37 R. Schweins, J. Hollmann and K. Huber, Polymer, 2003, 44, 7131-7141.

38 C. Heitz, M. Rawiso and J. Francois, Polymer, 1999, 40, 16371650.

39 Light Scattering from Polymer Solutions, ed. M. B. Huglin, Academic Press, New York, 1972.

40 A. Silberberg, J. Eliassaf and A. Katchalsky, J. Polym. Sci., 1957, 23, 259-284.

41 G. Savin and W. Burchard, Macromolecules, 2004, 37, 30053017.

42 W. Burchard, Macromolecules, 2004, 37, 3841-3849.

43 U. M. Apel, R. Hentschke and J. Helfrich, Macromolecules, 1995, 28, 1778-1785.

44 H. D. Burrows, D. Costa, M. L. Ramos, M. G. Miguel, M. H. Teixeira, A. A. C. C. Pais, A. J. M. Valente, M. Bastos and G. Bai, Phys. Chem. Chem. Phys., 2012, 14, 7950-7953.

45 S. Peljhan, E. Žagar, J. Cerkovnik and K. Kogej, J. Phys. Chem. B, 2009, 113, 2300-2309.

46 K. Buyse and H. Berghmans, Polymer, 2000, 41, 1045.

47 J. De Rudder, B. Berge and H. Berghmans, Macromol. Chem. Phys., 2002, 203, 2083.

48 B. Hess and N. F. A. van der Vegt, Proc. Natl. Acad. Sci. U. S. A., 2009, 106, 13296-13300. 